\title{
Autonomous 3D Target Tracking via UAV
}

\author{
Şeyma Sivritaş and Ahmet Tekin
}

\begin{abstract}
In recent years, unmanned aerial vehicles (UAVs) have been integrated into many applications. These vehicles are used by the governments and the private sector for many different purposes. Some of the popular applications are field scanning, target tracking, defense equipment, search and rescue, map creation, underground resource search, photography or entertainment. Recently many academic institutions have shown significant amount of interest in this field and many of them have established aviation departments and societies. In the recent years, many thesis studies and academic publications related to UAVs have been presented. The aim of this particular study is to detect a small object identified as a target by UAV and to provide autonomous tracking of the detected target in three axes. As the outcome of this work, the UAV is coded to lock on the target and to maintain a fixed distance on the $x, y$ and $z$ axes after the initial lock. Within the scope of the study, a functional system was designed by evaluating the most important parameters for UAVs such as weight, cost and flight time. An algorithm was designed that uses the image processing methods to detect the target and movement direction of the target. The system utilizes MAVLink communication protocol to control the UAV. In addition, two different tests and test results corresponding to two distinct methods were reported which were done to keep the distance constant on the z-axis by using an external distance sensor or comparison algorithm. The project was successfully implemented using single camera only.
\end{abstract}

Index Terms-Unmanned aerial vehicle, UAV, autonomous system, 3D tracking, image processing, fast algorithm.

\section{INTRODUCTION}

$\mathrm{I}$ N RECENT DECADES, unmanned aerial vehicles (UAVs) have become quite widespread and popular. These vehicles may be deployed for many different purposes. The main use cases for these technologies are field scanning, target detection, target tracking, attack, search and rescue, map creation, photography or entertainment. UAVs are also popular topic for academic research [1-3].

ŞEYMA SIVRITAŞ, is with Department of Electrical and Electronics Engineering, Ozyegin University, Istanbul, Turkey, (e-mail: seyma.sipar@gmail.com).

(iD https://orcid.org/0000-0001-5957-0890

AHMET TEKIN, is with Department of Electrical Engineering, Istanbul Technical University, Istanbul, Turkey, (e-mail: ahmettekin@itu.edu.tr).

iD https://orcid.org/0000-0001-8549-2582

Manuscript received Jun 22, 2020; accepted Dec 23, 2021.

DOI: $10.17694 /$ bajece. 756443
Tracking an object by a drone is widely implemented project utilizing multiple simultaneously operating sensors. In one of these projects, mainly two embedded cameras were used for object detection and tracking. An algorithm was used to provide tracking in the 3 th axis without a dedicated distance sensor [1].

There is another prominent work in the literature about autonomous flight. A reinforcement learning method was also applied in this work. In the particular system, a camera subsystem, which consists of 24 cameras, was used for this study [4].

In 2016, a group has reported a real time object tracking project and the project outcome was successfully demonstrated. In the particular project, a special motion capture camera system was used to detect the position of the moving object and Matlab Simulink was used to process the information. In addition, an external computer was used as a base station [5].

In another similar project, a visual detection and tracking implementation was realized with UAVs. In this project, Haar cascade classifier was used to detect the object and two cameras were used to implement the target locking function [6].

Within the scope of this work, a programmable autonomous UAV system was designed and a test mission was proven successfully. The mission was detection of a target object and 3D (3-dimensional) tracking (on $\mathrm{x}, \mathrm{y}, \mathrm{z}$ axes) of the target autonomously. In this paper, main components of the autonomous UAV system are listed and described. Also, design steps of the image processing subsystem were summarized. The method of detecting the position of the moving target and calculation of the updated coordinates according to displacement of the target were presented. Two different techniques for altitude tracking were analyzed and the results were compared.

\section{METhodology AND DESIGN}

Overall system consists of two main platforms; hardware and software platforms. The mission can be analyzed in three sub-sections to achieve autonomous 3D tracking task successfully.

\section{A. Hardware Platform of the System}

A UAV was used with SDK (Software Development Kit) flight controller to achieve the mission. Some auxiliary components (sensors) were also used to complete the autonomous system design. These components are: 
BALKAN JOURNAL OF ELECTRICAL \& COMPUTER ENGINEERING， Vol. 10, No. 1, January 2022

- GPS was used to determine the located coordinates of the UAV.

- Compass was used to determine the directions.

- Accelerometer was used to determine the acceleration in three axes $(\mathrm{x}, \mathrm{y}, \mathrm{z})$.

- Gyroscope was used to determine the motion in three axes $(\mathrm{x}, \mathrm{y}, \mathrm{z})$.

- Barometer was used to determine the altitude by using pressure value.

- On-board computer was used to provide the communication with the flight controller of the UAV for behavior commands. It was also used as hardware platform for image processing software.

- Camera was used to get the image for image processing.

- Distance sensor was used to provide altitude tracking.

\section{B. Software Platform of the System}

\section{1) Image Processing}

Image processing application was used for detection and tracking of the target. All image processing steps were implemented online with an on-board computer. Within the scope of image processing, Python programming language and OpenCV library were preferred.

Python is an interpreted programming language and doesn't need compiling process [7]. OpenCV is a library for programming languages. It is open source and very helpful. This library provides significant support in coding and hence saves time [8]. Both of Python and OpenCV are widely used in an image processing area [9].

\subsection{Color space}

The image is captured in RGB color space from camera but it is converted to HSV (Hue, Saturation, Value) color space to have more details about color such as saturation and light intensity. The most accurate HSV values can be obtained by experimentally masking the target in the mission environment $[10,11]$.

\subsection{Gaussian filtering (Blurring)}

Gaussian filtering is a kind of low-pass filter and this function is used to blur an image. This filter prevents sharp transition (high frequency) in the image and provides smoothing. Noise components can be attenuated with the help of this filter [8, 12].

\subsection{Masking}

The desired object is masked from whole image by making the color of the target object white and color of the rest of the image black. This process is necessary to implement Morphological Transformations to be described in next section to obtain better quality from captured image by filtering out the noise $[10,11]$.

\subsection{Erosion}

The Erode function is used for eroding boundaries of the objects and this function provides decreasing the area of the objects. The Erode function is very useful for removing noise. To protect the size of the original objects, the "Dilation" function is used after erosion process [13-16].

\subsection{Dilation}

The dilate function is used to expand the boundaries of the objects and this function provides enlarging the area of the objects. The Dilate function is generally used with "Erode" function, after erosion [13-16].

\section{2) Control Mechanism}

The stabilization of the UAV was provided with the help of PID control mechanism integrated into flight controller. PID (Proportional, Integral, and Derivative) controller which controls the process variables by using a control loop feedback mechanism. This system is used to minimize the error of the system dynamically. The difference between a desired set point and a measured process variable is calculated by PID controller. There are three parameters in PID controller calculation. The first component is proportional control; it controls the actual value of the error. The second component is integral control; it takes into account the error history. Finally, third component is derivative control, it acts based on the rate of error $[17,18]$. The block diagram of the PID controller is shown in Fig. 1 and the calculation method is shown in equation (1).

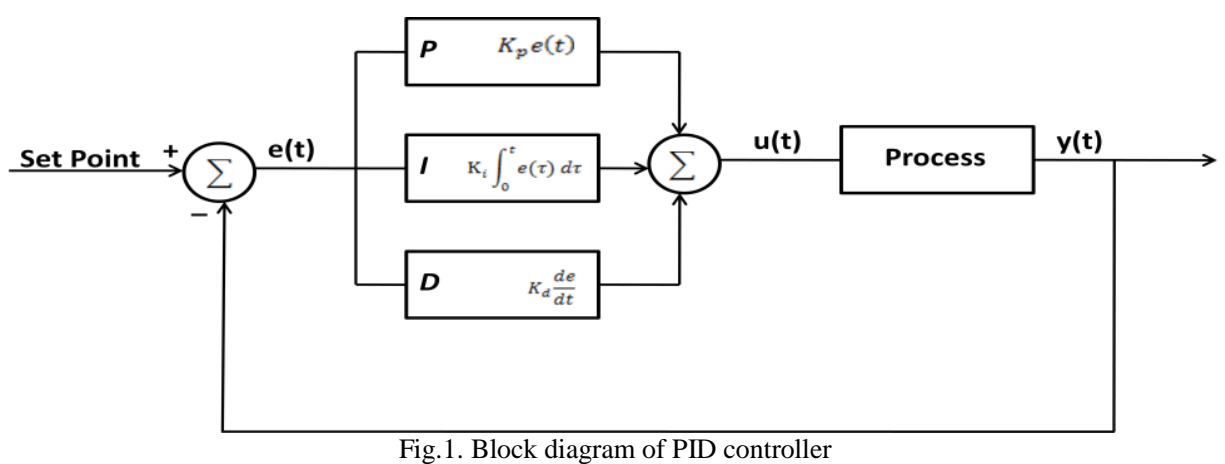


BALKAN JOURNAL OF ELECTRICAL \& COMPUTER ENGINEERING， Vol. 10, No. 1, January 2022

$u(t)=k_{p} e(t)+k_{i} \int_{0}^{t} e(\tau) d \tau+k_{d} \frac{d e}{d t}$

where,

Kp: Proportional gain

Ki: Integral gain

Kd: Derivative gain

u: Control signal

e: Error

\section{3) Communication Protocol}

MavLink (The Micro Air Vehicle Link) is one of the most popular communication protocols for Unmanned Aerial Vehicles. MAVLink protocol was used for communication with UAV and on-board computer. This protocol also can be used between UAVs and GCS (Ground Control Station) over a wireless channel [19].

\section{Design and Mission Steps}

Before starting the mission, the target was determined as a red point and a red ball with a diameter of $5 \mathrm{~cm}$ was used during the mission trials. The determined color was defined to the system with the HSV color space parameters. Since the color detection sensitivity of the system changes according to the ambient brightness, the appropriate color range was decided by experimenting. Detection of objects smaller than 3 pixels was inhibited to prevent unwanted pixel noise from being detected as a target. As a performance criterion, the system could detect and track a red point larger than 3 pixels which is within the capacity of the ultrasonic sensor. The mission can be divided into four parts to be able to analyze it in detail.

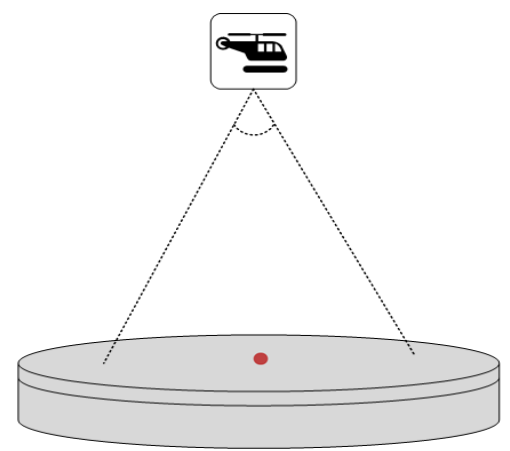

Fig.2. Illustration of angle of view

\section{1) Target Detection}

When the red point target is detected by the UAV, the UAV remains over the target. The resolution of the camera was set as $640 \times 480$ pixels in this case. This resolution values show that the horizontal area of the camera will be divided into 640 equal parts (pixels) and the vertical area will be divided into 480 equal parts (pixels). These horizontal and vertical areas are related to angle of view of the camera. Illustration of the angle of view can be seen from Fig. 2 .

The angle of view values of the used camera are as follows [20]:

Horizontal viewing angle: $53.50^{\circ}$

Vertical viewing angle: $41.41^{\circ}$

The field of view of the camera is calculated using angles of view of the camera with the help of cosine theorem. Illustration of the field of view is given in Fig. 3. Equation (2) and (3) can be used for these calculations.

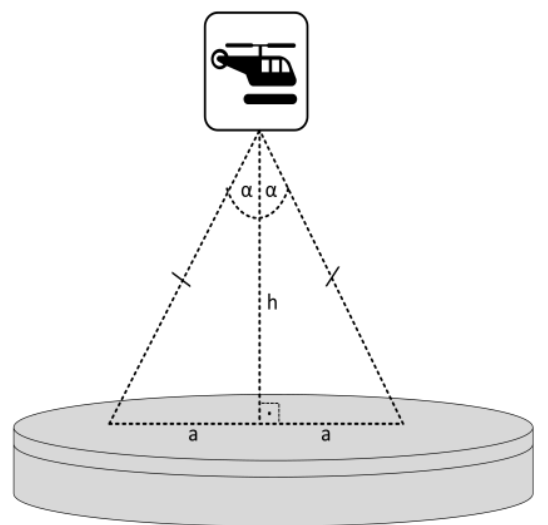

Fig.3. Illustration of field of view

$$
\begin{aligned}
& \frac{h}{\operatorname{Cos}(\alpha)}=\frac{a}{\operatorname{Sin}(\alpha)} \\
& a=h \frac{\operatorname{Sin}(\alpha)}{\operatorname{Cos}(\alpha)}
\end{aligned}
$$

In above equations, $h$ (altitude) is measured by the barometer and this data is taken from barometer output. The calculated field of view is divided by 640 for the horizontal axis and the length corresponding to a single pixel on the horizontal axis (x-axis) is obtained. Similarly, the calculated field of view of the vertical axis is divided by 480 and the length corresponding to a pixel on the vertical axis (y axis) is calculated. These calculations can be formulated as shown in equation (4) and (5).

$$
\begin{aligned}
& P_{h}=\frac{F_{h o V}}{640} \\
& P_{v}=\frac{F_{0 V}}{480}
\end{aligned}
$$

where,

$\mathrm{P}_{\mathrm{h}}$ : Unit pixel length on horizontal axis

$\mathrm{P}_{\mathrm{v}}$ : Unit pixel length on vertical axis

FoV : Field of view on horizontal axis 
FoV $_{\mathrm{v}}$ : Field of view on vertical axis

\section{2) Calculation of New Coordinate of Moving Target}

If the detected target moves out of the center of the camera, the autonomous system follows the target and gets the target position back to the center of the field of view. In order to do this, first, the new location of the detected target is determined. By comparing the pixel location at the center of the target with the pixel location at the center of the field of view, the target location can be obtained with respect to the center frame. Then the distance of the target from the center is calculated in meters by multiplying the pixel distance with pixel length. The illustration of this step can be seen from Fig. 4.

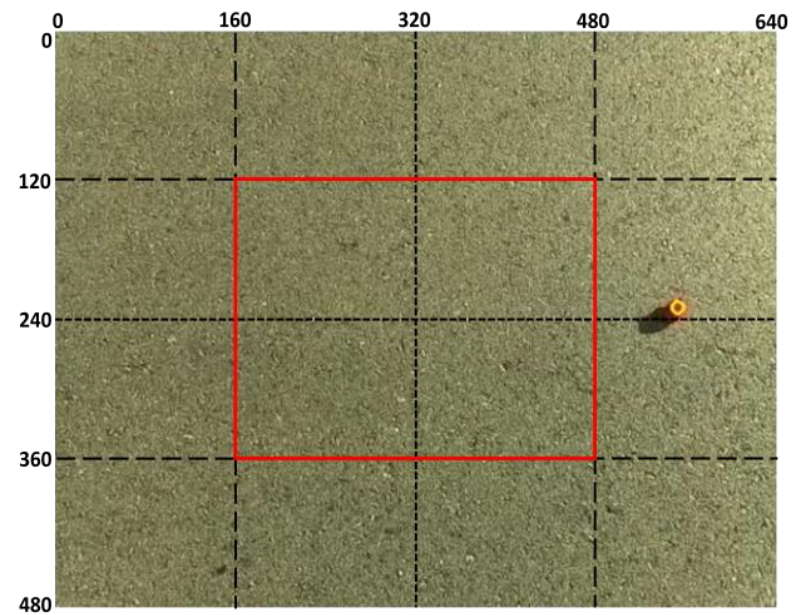

Fig. 4. Illustration of target pixel position

The center coordinate of the field of view is $(320,240)$ pixels on horizontal (x) and vertical (y) axes, respectively. The four sides (left, right, up, down) of the center were divided into two parts. The intersection points in all quarter parts specify the corner points of the tolerance frame. This frame is shown with red color in Figure 4. In this figure, target was detected at the pixel position of $(546,228)$ as seen from Fig. 5. Since the vertical $228^{\text {th }}$ pixel is inside the tolerance frame, movement on the vertical axis is not required. However, the horizontal $546^{\text {th }}$ pixel is out of tolerance frame, and the UAV should move to the right direction on the horizontal axis to make the target placed in the tolerance frame. General formulation to determine the required movement distance to get the target into center of the frame can be shown in the relation between Equations (6) and (9).

$\operatorname{Pd}_{\mathrm{h}}=320-\mathrm{x}$

$\operatorname{Pd}_{\mathrm{v}}=240-\mathrm{y}$

$\mathrm{M}_{\mathrm{h}}=\mathrm{Pd}_{\mathrm{h}} \times \mathrm{P}_{\mathrm{h}}$

$\mathrm{M}_{\mathrm{v}}=\mathrm{Pd}_{\mathrm{v}} \times \mathrm{P}_{\mathrm{v}}$

where,

$\mathrm{Pd}_{\mathrm{h}}$ : Pixel distance from center on horizontal axis
$\mathrm{Pd}_{\mathrm{v}}$ : Pixel distance from center on vertical axis

$\mathrm{M}_{\mathrm{h}}$ : Required movement distance on horizontal axis

$\mathrm{M}_{\mathrm{v}}$ : Required movement distance on vertical axis

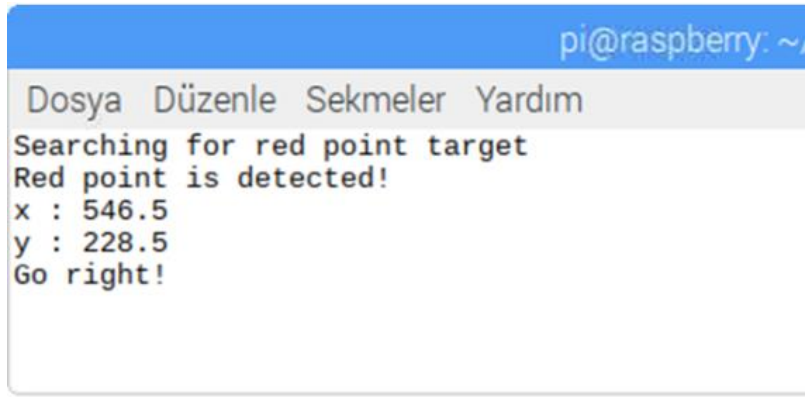

Fig. 5. Command output window for detection of target pixel position

Calculated distance data is converted to coordinate by using coordinate calculation method. Latitude angle and Longitude angle are used as coordinate parameters. These angles can be seen from Fig. 6. Equations (10) and (11) can be used for calculation of the angle difference [21]. Calculated angle difference values are then added to original angle values to obtain the new coordinates as seen from equation (12) and (13).

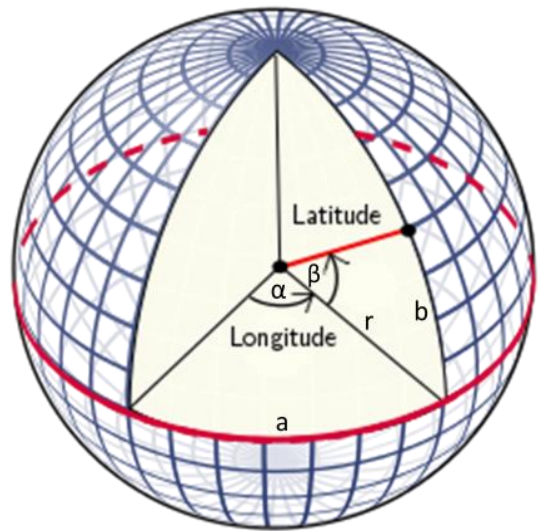

Fig. 6. Latitude and Longitude angles [22]

$\beta=\frac{b}{r}$

$\alpha=\frac{\mathrm{a}}{\mathrm{r} \operatorname{Cos} \beta}$

New Longitude angle $=\alpha_{\text {org }}+\alpha$

New Latitude angle $=\beta_{\text {org }}+\beta$

where,

$\alpha$ : Longitude angle difference (radian)

$\beta$ : Latitude angle difference (radian)

a: Displacement in latitude (m)

b: Displacement in longitude (m) 


\section{3) Moving to New Coordinate}

The calculated coordinate values are sent to the UAV via the MAVLink protocol and the UAV is made to move over the target. This part can be seen from "Results" section clearly.

\section{4) Altitude Tracking}

After the $\mathrm{x}$ and $\mathrm{y}$ axes were checked and the position of the target was brought to the center of the frame by the autonomous UAV, the third axis, $\mathrm{z}$ axis (height), is checked. Then, the current distance value is compared with the target distance and the UAV behaves according to the comparison result and reaches the required height. Two different techniques have been implemented within the scope of the study.

\subsection{Distance calculation with algorithm}

In the camera based distance calculation technique, single camera and a simple pixel algorithm are used. No other components are necessary to obtain the approximate distance.

In this technique, a change in the radius of the object detected as a target is observed. The current altitude and radius are recorded when the target was first detected. If the target moves up or down while the UAV is hovering at a constant altitude after detection, the measured radius will also change. This change will be positive if the object moves upwards and approaches the UAV, and if the object moves downwards and away from the UAV the change will be negative. After the trigonometric calculations, the new radius and the previous radius are compared to determine the amount and direction of change in distance. Calculation can be made by using triangle similarity theorems. This process can be understood with the help of Fig. 7, equation (14) and (15).

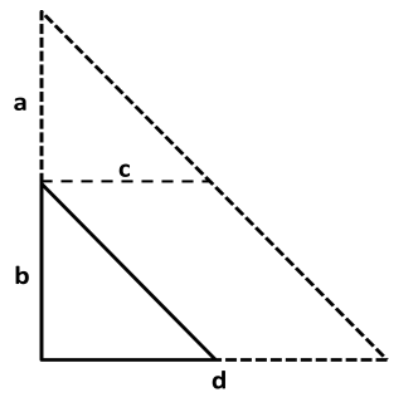

Fig. 7. Illustration of triangle similarity

$\frac{a}{a+b}=\frac{c}{d}$

Here, " $d$ " represents the radius of the object $\left(r_{e x}\right)$ and "a+b" represents the altitude whereas " $c$ " represents the length of the new radius $\left(\mathrm{r}_{\text {new }}\right)$ and "a" value represents the new altitude.

ratio $=\frac{r_{\text {new }}}{r_{\mathrm{ex}}}$

If ratio $>1$;

it is understood that the object is rising and approaching the UAV. The distance is calculated by multiplying the current height by calculated ratio. The obtained altitude value is sent to the UAV as an ascent command.

\section{If ratio $<1$,}

it is understood that the object is descending and moving away from UAV. The distance is calculated by multiplying the current height by calculated ratio. The obtained altitude value is sent to the UAV as a descend command.

There is a possible problem while calculating the distance with this technique. In some cases, even if the position of the object does not change, its radius can be measured smaller than it appears. As a result, since the autonomous UAV perceive that the object is farther from the UAV, the UAV may want to approach the object. The illustration of this relation can be seen from Fig. 8 .

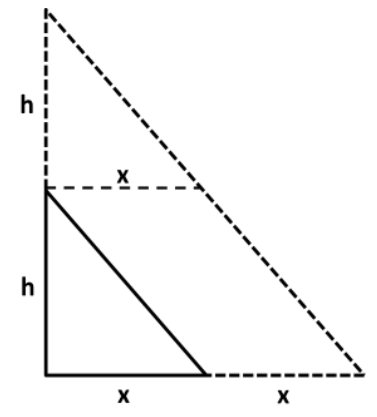

Fig. 8. Illustration of radius measurement

When the altitude of the UAV is " $2 \mathrm{~h}$ " and the radius of the target is " $2 x "$, if the radius of the object is measured as " $\mathrm{x}$ " instead of " $2 x$ ", the UAV wants to reduce the altitude by the same radius ratio. Thus, the UAV will move downwards until the altitude is "h". This type of system behavior is undesirable. The source of this problem may be some factors such as image quality, camera resolution, and variation of light intensity or change of light angle. Some examples of this kind of problem can be seen in Fig. 9 through Fig. 12.

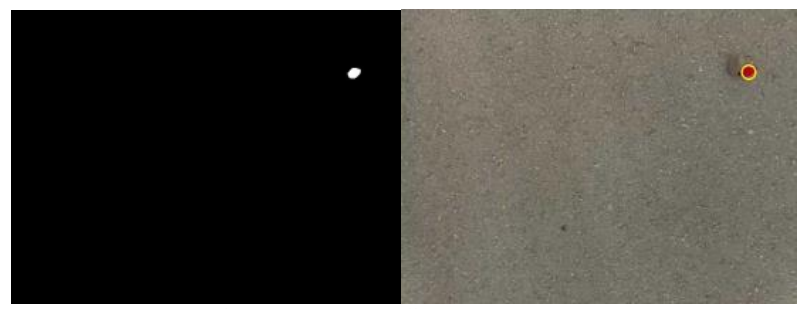

Fig. 9. Correct radius measurement

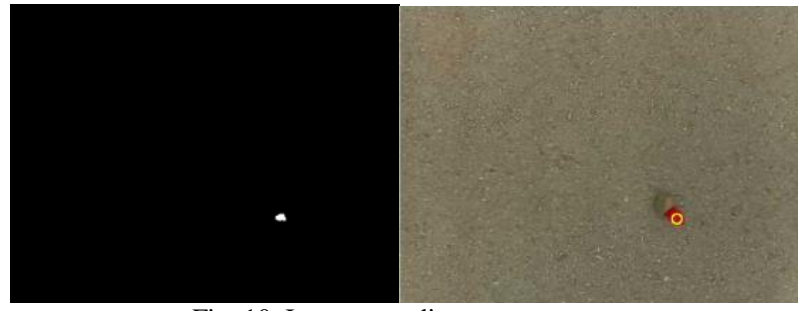

Fig. 10. Incorrect radius measurement 


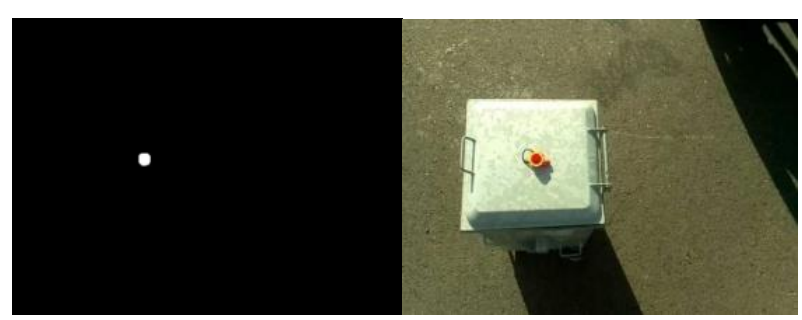

Fig. 11. Correct radius measurement

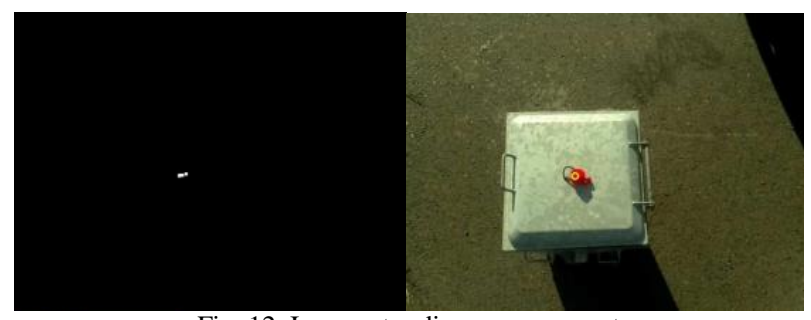

Fig. 12. Incorrect radius measurement

In this experiment, it was observed that the probability of obtaining an incorrect value as a result of the distance measurement made by the algorithm method is higher than the measurement method made while utilizing a precision distance sensor. Therefore, although the distance calculation method with the algorithm is lower in cost, lighter in weight and simpler in terms of coding, the mission is carried out by employing an additional distance sensor.

\subsection{Distance calculation with distance sensor}

Different distance sensors can be used in this method. Two commonly used distance sensors are LIDAR and Ultrasonic sensors.

\subsubsection{Lidar sensor}

Laser technology is used in LIDAR (Light Detection and Ranging) measurement sensor [23]. The single-beam narrowband laser is generated and then it is reflected from target object [24]. The illustration of the working principle of Lidar sensor is shown in Figure 13. The distance can be calculated by the half of duration when the laser pulse being emitted and the time when the reflected laser pulse being sensed. The distance can be calculated by multiplying the half of the total travel duration by the speed of light $(299,792,458 \mathrm{~m} / \mathrm{s})$. The result will be in meters and the formula can be seen from equation (16) [25].

$\mathrm{D}=\mathrm{t} \cdot \mathrm{c}$

where,

\section{D: Distance from object (m)}

$\mathrm{t}$ : Half of time interval between generated and reflected laser pulse

c: speed of light $(299,792,458 \mathrm{~m} / \mathrm{s})$

\subsubsection{Ultrasonic sensor}

Sound wave technology is used for this type of distance sensor. The sensor generates $40 \mathrm{kHz}$ sound pulse when it is triggered then waits and listens to reflected sound wave. Therefore, the total travel time $(2 \mathrm{t})$ of the sound wave can be measured as transmitted and reflected duration [26]. The illustration of the working principle of ultrasonic sensor is shown in Fig. 14. The distance can be calculated by multiplying the half of the total travel duration by the speed of the sound wave $(343 \mathrm{~m} / \mathrm{s})$ [27]. The result will be in centimeters and the formula can be seen from equation 17 .

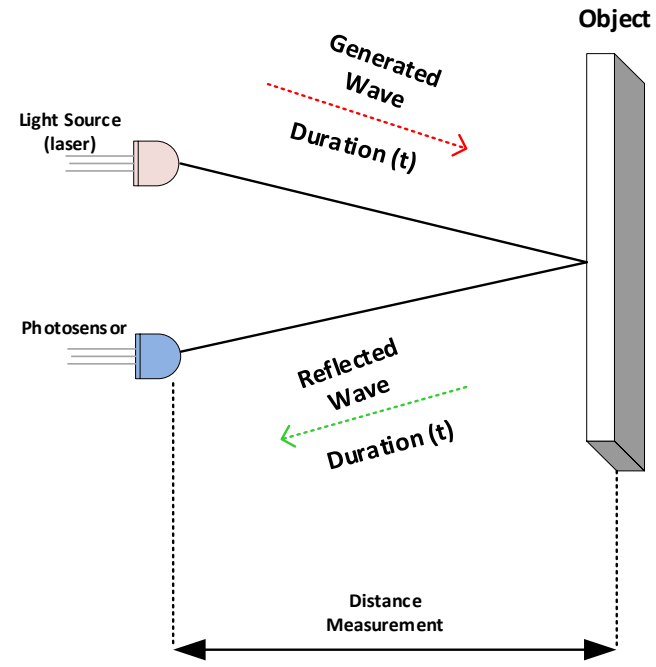

Fig. 13. Working principle of Lidar Sensor

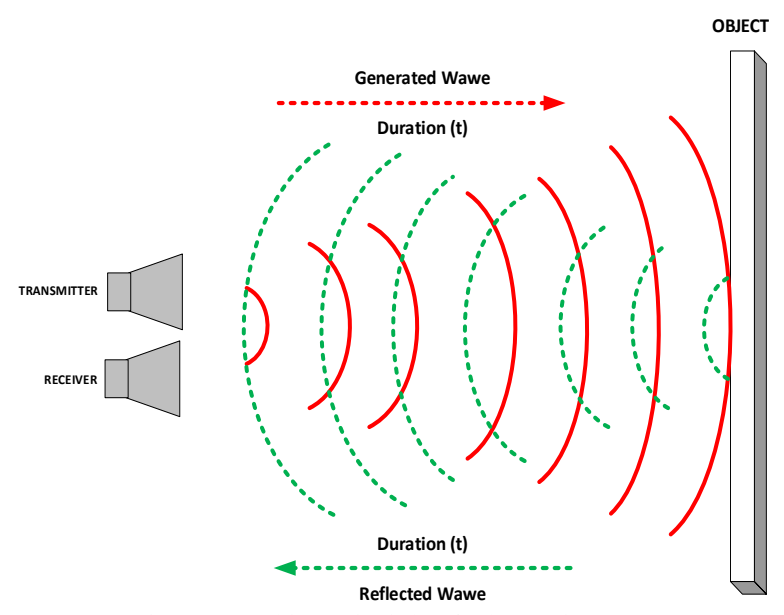

Fig. 14. Working principle of Ultrasonic Sensor

$\mathrm{D}=\mathrm{t} \cdot 34300$

where,

D: Distance from object $(\mathrm{cm})$

$\mathrm{t}$ : Half of time interval between generated and reflected sound wave

From these two options, using LIDAR is high in terms of cost and hence, it was preferred to use an ultrasonic sensor in the scope of the study. The range of the chosen ultrasonic sensor is $400 \mathrm{~cm}$.

The ultrasonic sensor is located very close point to the lens of the camera used for image processing. The sensor thus calculates the distance corresponding to the center of the field 
of view. The distance from the target at the time the target was first detected during the task is recorded as the "target distance". A hysteresis loop-style control mechanism was integrated to prevent the oscillation because of minor measurement error and small swings of the drone. In this context, a tolerance of $10 \%$ was allowed for distance change. That is, if the object is between $0.9 \mathrm{x}$ target distance and $1.1 \mathrm{x}$ target distance, the UAV will continue to maintain its current position. If the height of the target changes more than the specified tolerance, the result obtained by sensor measurement is compared with the first value recorded, target distance. If the difference is smaller than the target distance, it is understood that the object rises and approaches the UAV. The new altitude is calculated by adding the obtained difference with the current altitude. The calculated altitude value is sent to the UAV as an ascent command. If the difference is greater than the target distance, it is understood that the object is lowered away from the UAV. The new altitude is calculated by subtracting the obtained difference from the current altitude. The calculated altitude value is sent to the UAV as a descend command.

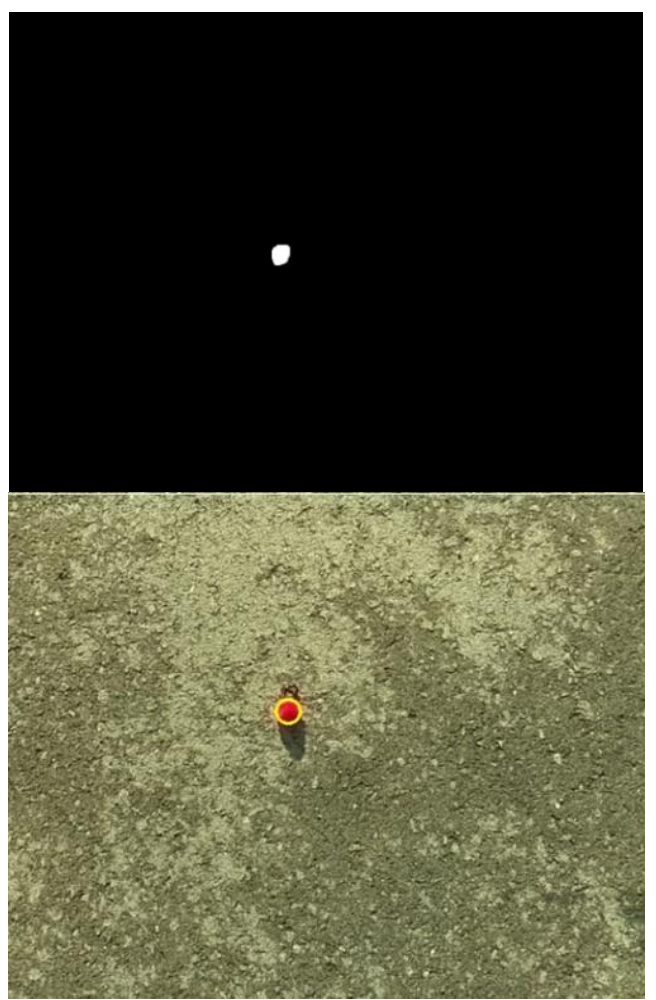

Fig. 15. Detection of the target

This process can be represented with the following equations (18) and (19). Altitude tracking photos can be seen from Fig. 15 to Fig. 17.

$\mathrm{D}=\mathrm{C}-\mathrm{T}$

$\mathrm{N}=\mathrm{A}-\mathrm{D}$

where,

A: Current Altitude (obtained from barometer) C: Current Distance. The current distance between UAV and target (obtained from ultrasonic sensor).

D: Difference from target distance
$\mathrm{N}$ : New Altitude

T: Target Distance. The distance between UAV and target at first target detection (constant, obtained from ultrasonic sensor).

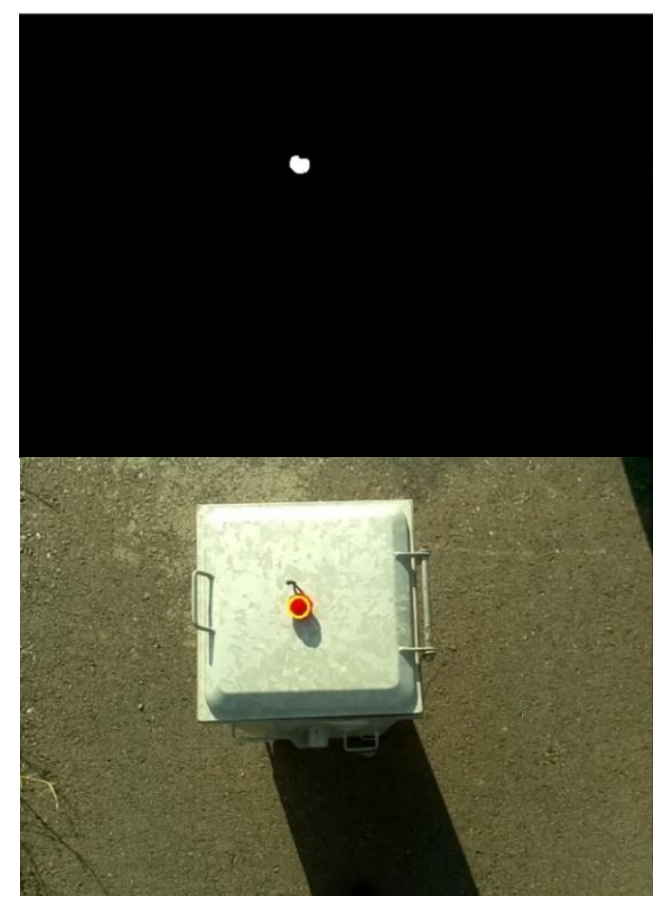

Fig. 16. Altitude tracking of the target

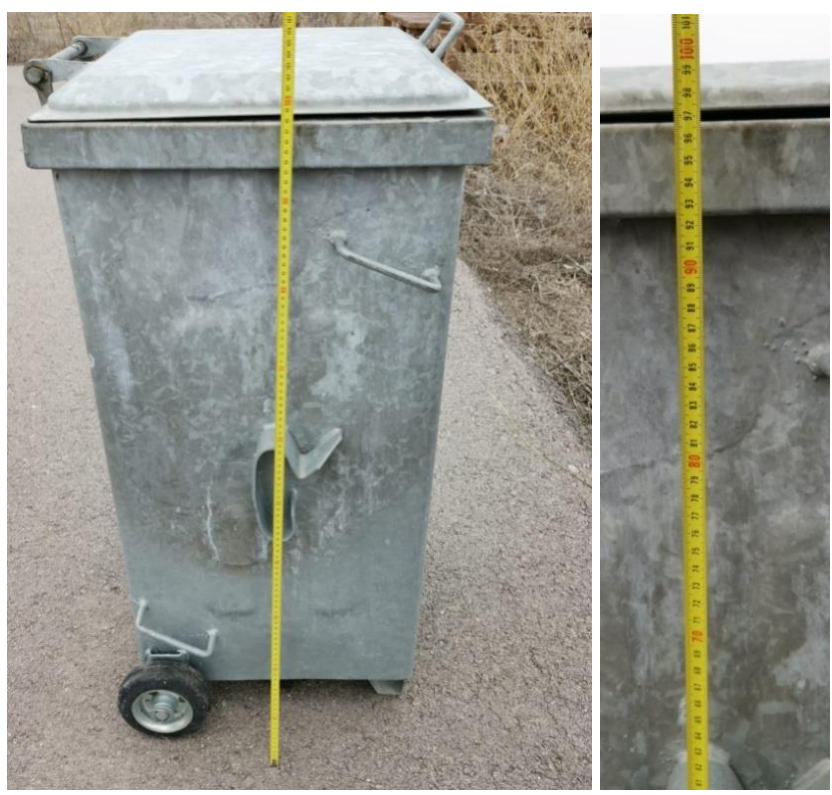

Fig. 17. Measurement of displacement in z-axis (height)

\section{RESULTS}

A simple mission is illustrated in Fig. 18 and tracking commands are given as shown in Fig. 19. The command window output was simplified to reduce the amount of writing and facilitate intelligibility. The basic functional behavior of the autonomous UAV system can be observed from the test track shown in Fig. 18. This figure is taken from GCS and the UAV was illustrated as green colored quad-copter on this satellite street view. The UAV was taken off from Home 
position (represented by $\mathrm{H}$ letter with green color). While searching the field, a target was detected at the point shown as "Tracking is Starting" with yellow color. Then the related commands were sent to the flight controller by on-board computer according to movement of the target. Movement positions were drawn by ground control station with purple line and directions were shown with red arrows. Commands were added on the figure with red color as well. At point "A", the target was placed 1 meter above the ground and this difference was detected by UAV as seen from the command window.

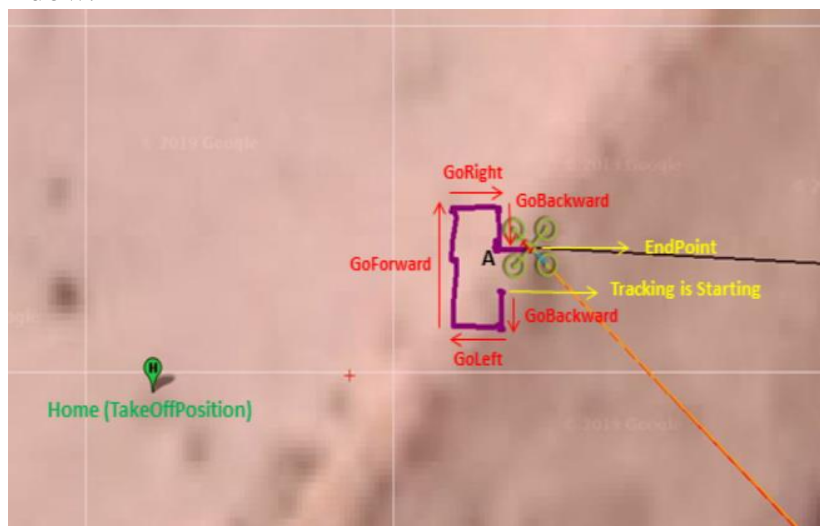

Fig. 18. Illustration of 3D tracking mission

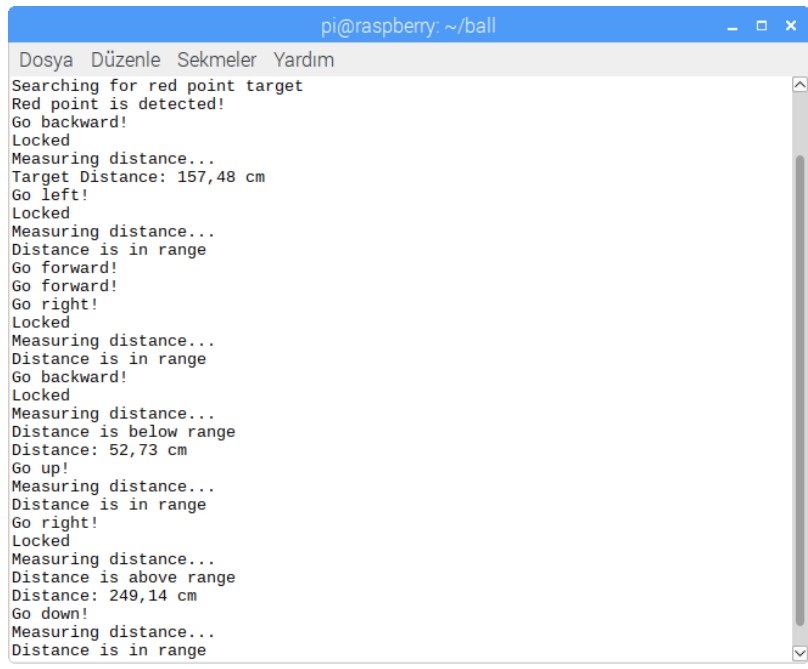

Fig. 19. Command output window of 3D tracking mission

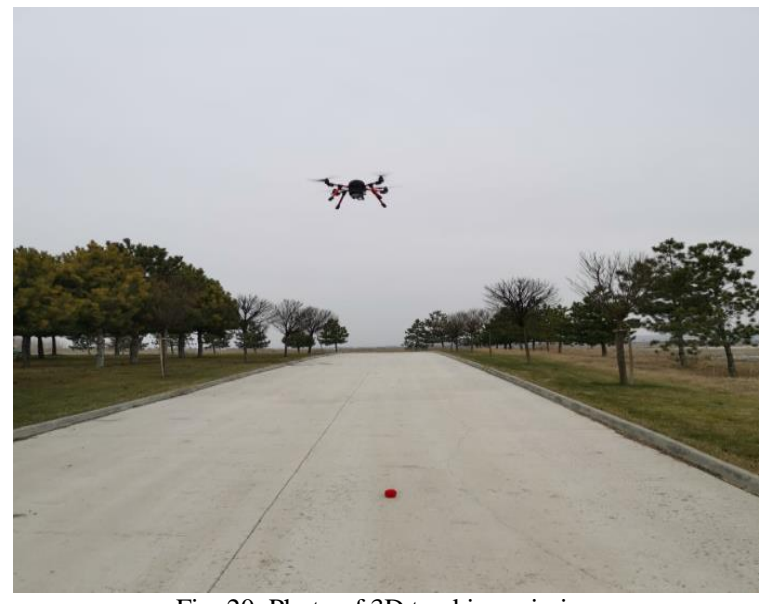

Fig. 20. Photo of 3D tracking mission
Finally, target was placed in the previous position again and the UAV was moved down until it reached target distance as seen in the command window. At this point, the mission was complete and the point was marked as "End Point" and indicated by the yellow arrow. The photo of the autonomous $3 \mathrm{D}$ target tracking mission in action is shown in Fig. 20.

\section{DISCUSSION AND CONCLUSION}

A target object can be detected and can be tracked on all three axes (x, y, $\mathrm{z}$ axes) with the aid of a UAV, an image processing system and a distance sensor. The distances to be moved for right-left ( $\mathrm{x}$ axis/roll) and forward-backward directions ( $\mathrm{y}$ axis/pitch) were calculated by using coordinate calculation methods, subsequently they are sent to flight controller and the UAV reached the target points successfully. It has been observed that using a single camera and basic distance algorithm option for tracking altitude ( $\mathrm{z}$ axis) is not a good enough solution to get stable results due to environmental factors and systemic offsets in the camera sensor. The mission of three-dimensional ( $\mathrm{x}, \mathrm{y}, \mathrm{z}$ axes) tracking of an object was successfully completed using a single camera, an additional distance sensor and a simpler algorithm. All project components were used by taking into account factors such as cost, weight and ease of application. Such a system can be customized for various tracking applications. The designed UAV can be used for applications such as; entertainment, scanning and tracking, with an even more attractive use case in the defense markets. If an object with complex boundaries is desired to be detected, it will be a little more complex and difficult to define these boundaries clearly, but, progressively smarter algorithms can enable these applications in the near future. The system can be improved significantly by using additional long-range Lidar sensors and high-resolution cameras for applications where the cost is not as important concern. Thus, tracking tasks can be performed more accurately and precisely with such an upgraded system.

\section{REFERENCES}

[1] R. Barták, A. Vyškovský, "Any Object Tracking and Following by a Flying Drone." Fourteenth Mexican International Conference on Artificial Intelligence. Cuernavaca, US, 2015.

[2] A. Hinas, J.M. Roberts, F. Gonzales, "Vision-Based Target Finding and Inspection of a Ground Target Using a Multirotor UAV System", Sensors, vol. 17, no.12, 2017, pp. 1-17.

[3] A. Audi, M. Pierrot-Deseilligny, C. Meynard, C. Thom, "Implementation of an IMU Aided Image Stacking Algorithm in a Digital Camera for Unmanned Aerial Vehicles", Sensors, vol. 17, no.7, 2017, pp. 1-21.

[4] J. Junell, E.J. Kampen, C. Visser, Q. Chu, "Reinforcement Learning Applied to a Quadrotor Guidance Law in Autonomous Flight." AIAA Guidance, Navigation, and Control Conference, Florida, US, 2015.

[5] A. Mashood, A. Dirir, M. Hussein, H. Noura, F. Awwad, "Quadrotor Object Tracking using Real-Time Motion Sensing." 5th International Conference on Electronic Devices, Systems, and Applications, Ras Al Khaimah, United Arab Emirates, December 2016.

[6] D. A. Mercado-Ravell, P. Castillo and R. Lozano, "Visual Detection and Tracking with UAVs, Following a Mobile Object", Advanced Robotics, vol.33, pp. 1-15, 2019.

[7] M. Lutz, Learning Python, O'Reilly Media, 2007, California, pp.700.

[8] S. Brahmbhatt, Practical OpenCV, Apress, USA, pp.223.

[9] R. Chityala, S. Pudipeddi, Image Processing and Acquisition using Python, CRC Press, Florida, pp.350.

[10] A. Mordvintsev, K. Abid, Changing Colorspaces. Accessed: Jul. 2019. [Online]. Available: 
https://opencv-pythontutroals.readthedocs.io/en/latest/py_tutorials/py_imgproc/py_colorspac es/py_colorspaces.html

[11] OpenCV, Thresholding Operations using inRange. Accessed: Jul. 2019. [Online]. Available: https://docs.opencv.org/3.4/da/d97/tutorial_threshold_inRange.html

[12] OpenCV, Smoothing Images. Accessed: Jul. 2019. [Online]. Available: https://docs.opencv.org/3.4.2/d4/d13/tutorial_py_filtering.html

[13] A. Koschan, M. Abidi, Digital Color Image Processing, John Wiley \& Sons, Inc., New Jersey, US, pp.375.

[14] B. Jähne, Digital Image Processing, 6th, revised and extended edition, 2005, Springer, Berlin, Germany, pp.607.

[15] W.K. Pratt, Digital Image Processing: PIKS Scientific inside, 4th edition, 2001, John Wiley\&Sons, Inc., New Jersey, US, pp.807.

[16] OpenCV, Morphological Transformations. Accessed: Aug. 2019. [Online]. Available:

https://docs.opencv.org/3.4.2/d9/d61/tutorial_py_morphological_ops.ht $\mathrm{ml}$

[17] R. Gamasu, V.R.B. Jasti, "Robust Cohen-Coon PID Controller for Flexibility of Double Link Manipulator", International Journal of Control and Automation, vol. 7, no.1, 2014, pp. 357-368.

[18] K.J. Åström, T. Hägglund, PID Controllers: Theory, Design, and Tuning, 2nd edition, Instrument Society of America, 1995, USA, pp.338.

[19] S. Atoev, K. Kwon, S. Lee, K. Moon, "Data Analysis of the MAVLink Communication Protocol." International Conference on Information Science and Communications Technologies. Tashkent, Uzbekistan, 2017.

[20] Raspberry pi, Camera Module. Accessed: May. 2019. [Online]. Available: https://www.raspberrypi.org/documentation/hardware/camera/

[21] J. Case, Astro Navigation Demystified, Revised edition, Bookcase Learning Resources, 2016, Weymouth, UK, pp. 324.

[22] A. Singh, Latitude and Longitude are angles, not just some numbers on the earth's surface. Accessed: Aug. 2019. [Online]. Available: https://medium.com/@atinders/latitude-and-longitude-are-angles-notjust-some-numbers-on-the-earths-surface-d16019aaf24a

[23] J. Laconte, S. Deschênes, M. Labussière, F. Pomerleau, "Lidar Measurement Bias Estimation via Return Waveform Modelling in a Context of 3D Mapping." International Conference on Robotics and Automation. Montreal, Canada, 2019.

[24] X. Li, Y. Guo, "Application of LiDAR Technology in Power Line Inspection." International Conference on Advanced Materials, Intelligent Manufacturing and Automation. China, 2018.

[25] G. Duh. Light Detection and Ranging (LiDAR), Lecture notes. Portland State University.

[26] N.A. Latha, B. R. Murthy, K. B. Kumar, "Distance Sensing with Ultrasonic Sensor and Arduino", International Journal of Advance Research, Ideas and Innovations in Technology, vol. 2, no.5, 2016, pp. $1-5$.

[27] A. Pantano, D. Cerniglia, "Simulation of laser-generated ultrasonic wave propagation in solid media and air with application to NDE", Applied Physics A, vol. 98, no.2, 2010, pp. 327-336.

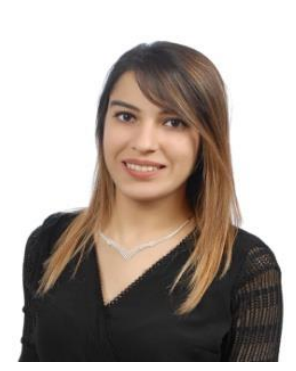

\section{BIOGRAPHIES}

ŞEYMA SIVRITAŞ received her B.Sc. degree of Electrical and Electronics Engineering from Dokuz Eylül University, İzmir, Turkey in 2015. She worked at Vestel Electronics between 2015 and 2018. She received her M.Sc. degree in the department of Electrical and Electronics Engineering from Özyeğin University, İstanbul, Turkey in 2020. She has been working at Turkish Aerospace as an Avionics System Engineer since 2018. She has experience in aircraft avionics systems design, power distribution system design and analysis of communication systems.

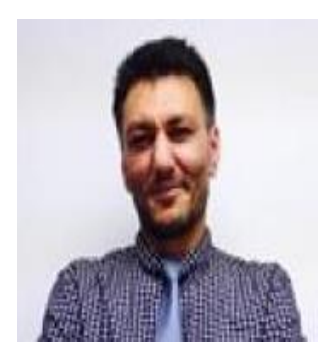

AHMET TEKIN has received his EE $\mathrm{PhD}$ degree from University of California Santa Cruz, CA, EE MS degree from North Carolina A\&T State University, Greensboro, NC and EE BS degree from Bogazici University, Istanbul, Turkey in 2008, 2004, and 2002, respectively. In addition to academic research in microelectronics, he worked for multiple innovative semiconductor design companies such as; Multigig, Inc., Newport Media, Aydeekay LLC, Broadcom corp., Semtech Corp., Nuvoton Technology Corp., Qualcomm and Waveworks Inc., leading semiconductor designs for communications, consumer and medical markets. His main focus area is analog/RF/mixed-signal integrated circuit design for communication and biomedical applications. $\mathrm{He}$ is currently engineering faculty member of Istanbul Technical University and serves in the board of directors of Waveworks Inc, Mission Viejo, CA. 